\title{
Correction: Dong, F.; et al. Disparities in Hypertension Prevalence, Awareness, Treatment and Control between Bouyei and Han: Results from a Bi-Ethnic Health Survey in Developing Regions from South China. Int. J. Environ. Res. Public Health 2016, 13, 233
}

\author{
Fen Dong ${ }^{1}$, Dingming Wang ${ }^{2}$, Li Pan ${ }^{1}$, Yangwen $\mathrm{Yu}^{2}{ }^{2}$ Ke Wang ${ }^{1}$, Ling Li ${ }^{2}$, Li Wang ${ }^{1}$, Tao Liu ${ }^{2}$, \\ Xianjia Zeng ${ }^{1}$, Liangxian Sun ${ }^{2}$, Guangin Zhu ${ }^{1}$, Kui Feng ${ }^{1}$, Biao Zhang ${ }^{1}, \mathrm{Ke} \mathrm{Xu}^{3}{ }^{3}$, Xinglong Pang ${ }^{3}$, \\ Ting Chen ${ }^{4}$, Hui Pan ${ }^{3}$, Jin Ma ${ }^{4}$, Yong Zhong ${ }^{4}$, Bo Ping ${ }^{5}$ and Guangliang Shan ${ }^{1, *}$ \\ 1 Department of Epidemiology and Biostatistics, Institute of Basic Medical Sciences Chinese Academy of \\ Medical Sciences, School of Basic Medicine Peking Union Medical College, Beijing 100005, China; \\ fionarab@163.com (F.D.); panli1716@163.com (L.P.); wangkehope@126.com (K.W.); \\ wangli0528@vip.sina.com (L.W.); zxj28@sohu.com (X.Z.); zhuguangjinpumc@126.com (G.Z.); \\ fengkui@sina.com (K.F.); sljzhangbiao11@126.com (B.Z.) \\ 2 Guizhou Center for Disease Control and Prevention, Guizhou 550004, China; \\ wangdingm123@sina.com (D.W.); yuyangweny@163.com (Y.Y.); gpicdp@163.com (L.L.); \\ liutao9099@163.com (T.L.); slx1087@163.com (L.S.) \\ 3 Department of Endocrinology, Peking Union Medical College Hospital, \\ Chinese Academy of Medical Sciences \& Peking Union Medical College, Beijing 100730, China; \\ zjwzxuke@126.com (K.X.); px119870610@163.com (X.P.); panhui20111111@163.com (H.P.) \\ 4 Department of Ophthalmology, Peking Union Medical College Hospital, \\ Chinese Academy of Medical Sciences \& Peking Union Medical College, Beijing 100730, China; \\ ct19870629@hotmail.com (T.C.); majin1912@163.com (J.M.); yzhong_eye@163.com (Y.Z.) \\ 5 Longli Center for Disease Control and Prevention, Guizhou 551200, China; gzlljkpb@126.com \\ * Correspondence: guangliang_shan@sohu.com; Tel.: +86-10-6915-5936
}

Academic Editor: Paul B. Tchounwou

Received: 5 August 2016; Accepted: 12 September 2016; Published: 17 September 2016

The authors wish to add the following amendments and corrections to their paper published in the International Journal of Environmental Research and Public Health [1].

Page 7, Section 3.3. Associated factors of hypertension: the odds ratio (OR) and $95 \%$ confidence interval (CI) for non/ex-drinkers and light drinkers are not consistent with the correspondent numbers in Table 3. The correct OR and 95\% CI should be:

\subsection{Associated Factors of Hypertension}

In multilevel logistic analyses of risk factors of hypertension, older age and comorbidities (central obesity, diabetes, dyslipidemia, or hyperuricemia) were associated with an increased risk of hypertension in both ethnic groups. In the Bouyei, non/ex-drinkers or light drinkers experienced significantly reduced risk than the harmful drinkers (OR 0.55, 95\% CI 0.42-0.72 for non/ex-drinkers; and OR $0.56,95 \%$ CI $0.41-0.77$ for light drinkers). In the Han, better education and having no family history of hypertension were observed to be associated with a decreased risk of hypertension (Table 3). 
Table 3. Results of Multilevel Logistic Models for Hypertension in Bouyei and Han.

\begin{tabular}{|c|c|c|c|c|}
\hline \multirow{2}{*}{ Variables } & \multicolumn{2}{|c|}{ Bouyei } & \multicolumn{2}{|l|}{ Han } \\
\hline & OR $(95 \% \mathrm{CI})$ & $p$ & OR $(95 \% \mathrm{CI})$ & $p$ \\
\hline Location $($ Ref $=$ rural $)$ & $0.99(0.63,1.56)$ & 0.9647 & $1.08(0.75,1.56)$ & 0.6750 \\
\hline Age (Ref $\leq 50$ years) & $5.23(4.11,6.65)$ & $<0.0001$ & $4.93(3.91,6.22)$ & $<0.0001$ \\
\hline Education $($ Ref $=$ low $)$ & - & - & - & - \\
\hline High & $0.79(0.50,1.24)$ & 0.2985 & $0.64(0.46,0.90)$ & 0.0096 \\
\hline Medium & $0.85(0.66,1.11)$ & 0.2292 & $0.74(0.57,0.96)$ & 0.0229 \\
\hline Insurance $($ Ref = no) & $0.75(0.26,2.15)$ & 0.5963 & $2.18(0.89,5.38)$ & 0.0900 \\
\hline Family history (Ref = yes) & $0.89(0.64,1.24)$ & 0.4795 & $0.57(0.46,0.71)$ & $<0.0001$ \\
\hline Physical activity $($ Ref $=$ low $)$ & - & - & - & - \\
\hline High & $1.01(0.79,1.29)$ & 0.9514 & $1.17(0.92,1.48)$ & 0.1953 \\
\hline Moderate & $0.84(0.56,1.26)$ & 0.3899 & $1.00(0.75,1.33)$ & 0.9723 \\
\hline Alcohol drinker $($ Ref = harmful $)$ & - & - & - & - \\
\hline Non/ex drinker & $0.55(0.42,0.72)$ & $<0.0001$ & $0.75(0.54,1.03)$ & 0.0805 \\
\hline Light drinker & $0.56(0.41,0.77)$ & 0.0004 & $0.77(0.54,1.08)$ & 0.1315 \\
\hline Smoking $($ Ref $=$ ever $)$ & $1.00(0.78,1.28)$ & 0.9886 & $0.89(0.70,1.12)$ & 0.3171 \\
\hline Central obesity $(\operatorname{Ref}=$ no $)$ & $1.96(1.49,2.58)$ & $<0.0001$ & $2.31(1.87,2.86)$ & $<0.0001$ \\
\hline Diabetes $(\operatorname{Ref}=\text { no })^{a}$ & $1.81(1.00,3.27)$ & 0.0489 & $2.08(1.42,3.04)$ & 0.0002 \\
\hline Dyslipidemia $(\operatorname{Ref}=\text { no })^{b}$ & $1.71(1.33,2.21)$ & $<0.0001$ & $1.32(1.07,1.65)$ & 0.0117 \\
\hline Hyperuricemia $(\operatorname{Ref}=$ no $)$ & $2.19(1.57,3.05)$ & $<0.0001$ & $1.98(1.49,2.63)$ & $<0.0001$ \\
\hline
\end{tabular}

Ref indicates reference. All the variables were included as fixed effects in multilevel models; ${ }^{\text {a }}$ Diabetes was defined as fasting glucose $\geq 7.0 \mathrm{mmol} / \mathrm{L}$ or reported diagnosis; $\mathrm{b}$ Dyslipidemia was defined as $\mathrm{TC} \geq 6.22 \mathrm{mmol} / \mathrm{L}, \mathrm{LDL} \geq 4.14 \mathrm{mmol} / \mathrm{L}, \mathrm{TG} \geq 2.26 \mathrm{mmol} / \mathrm{L}$, or $\mathrm{HDL}<1.04 \mathrm{mmol} / \mathrm{L}$.

The authors would like to apologize for any inconvenience caused to the readers by these changes.

\section{Reference}

1. Dong, F.; Wang, D.; Pan, L.; Yu, Y.; Wang, K.; Li, L.; Wang, L.; Liu, T.; Zeng, X.; Sun, L.; et al. Disparities in Hypertension Prevalence, Awareness, Treatment and Control between Bouyei and Han: Results from a Bi-Ethnic Health Survey in Developing Regions from South China. Int. J. Environ. Res. Public Health 2016, 13, 233. [CrossRef] [PubMed]

(C) 2016 by the authors; licensee MDPI, Basel, Switzerland. This article is an open access article distributed under the terms and conditions of the Creative Commons Attribution (CC-BY) license (http://creativecommons.org/licenses/by/4.0/). 